\title{
Farmacocinética de posaconazol en la profilaxis y tratamiento de la infección fúngica invasora en niños inmunocomprometidos en un hospital pediátrico
}

\author{
Romina Valenzuela, Patricio García, Marlon Barraza, Julia Palma, Paula Catalán, \\ M. Elena Santolaya, J. Pablo Torres y Jorge Morales
}

Pharmacokinetics of posaconazol in the prophylaxis and treatment of invasive fungal infection in immunocompromised children in a pediatric hospital

Background: There is no consensus on the optimal dosage use of posaconazole (PSC) for invasive fungal infection (IFI) in pediatric patients and normally it is adjusted with drug levels (DLs) $\geq 0.7 \mu \mathrm{g} / \mathrm{ml}$ and $\geq 1.25 \mu \mathrm{g} / \mathrm{ml}$ for prophylaxis and treatment, respectively. Objective: To describe the experience of monitoring DLs of PSC in immunocompromised pediatric patients with IFI and to determine if the recommended doses reach CP effective in prophylaxis $(\geq 0.7 \mu \mathrm{g} / \mathrm{mL})$ and treatment $(\geq 1.25 \mu \mathrm{g} / \mathrm{mL})$. Method: A retrospective analysis in children who received PSC from January 2012 to October 2016, in the Oncology and Bone Marrow Transplant units at Hospital Calvo Mackenna was done. Results: Six patients with 78 DLs were reviewed (4 prophylaxis and 4 treatment). Median PSC dose was 12.5 and $18.8 \mathrm{mg} / \mathrm{kg} / \mathrm{d}$ for prophylaxis and treatment, resulting in mean DLs of $0.97 \mathrm{and} 1.8 \mu \mathrm{g} /$ $\mathrm{mL}$ respectively. In prophylaxis $40 / 67(60 \%)$ were recorded with DLs $\geq 0.70 \mu \mathrm{g} / \mathrm{mL}$ receiving a median dose of $12.5 \mathrm{mg} / \mathrm{kg} / \mathrm{d}$. While for treatment: $5 / 11(46 \%)$ presented DLs $\geq 1.25 \mu \mathrm{g} / \mathrm{mL}$, receiving a median dose of $18 \mathrm{mg} /$ $\mathrm{kg} / \mathrm{d}$. Conclusion: Our results are in line with the recommended for PSC dosage, but individualized monitoring is required to maintain adequate DLs.

Key words: Posaconazole, drug levels, therapeutic drug monitoring, immunocompromised patients, children.

Palabras clave: Posaconazol, concentraciones plasmáticas, monitorización terapéutica de fármacos, pacientes inmunocomprometidos, niños.

\section{Introducción}

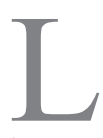

a infección fúngica invasora (IFI), es una complicación relevante en pacientes con enfermedades hematológicas y con trasplante de progenitores hematopoyéticos (TPH), dada la alta morbimortalidad causada por levaduras y hongos filamentosos. La tasa de mortalidad media relacionada con la candidiasis invasora (CI) es superior a $30 \%$ y para aspergilosis invasora (AI) mayor a $50 \%$ en algunos casos. El tratamiento de la IFI en estos pacientes es complejo, con la aparición de nuevos fármacos y pruebas diagnósticas que han dado lugar a diferentes estrategias terapéuticas, ya sea en forma profiláctica, terapéutica o empírica ${ }^{1-4}$. Una adecuada evaluación del riesgo individual de adquirir una IFI por cada paciente es crítica para elegir el mejor método para profilaxis o tratamiento, aumentando así las tasas de supervivencia. Entre los antifúngicos disponibles destacan los azoles: fluconazol, itraconazol, voriconazol y posaconazol (PSC), para la cobertura de levaduras y hongos filamentosos ${ }^{1-3}$.

Posaconazol es un antifúngico triazólico de amplio espectro, indicado para el tratamiento de infecciones por Aspergillus, Candida y Mucorales y disponible en nuestro medio como suspensión oral. La dosis profiláctica recomendada en adolescentes ( $>12$ años) y adultos es de $200 \mathrm{mg}$ cada $8 \mathrm{~h}$. También se ha visto una buena respuesta con dosis de tratamiento para IFI en adolescentes (> 12 años) y adultos, de $800 \mathrm{mg}$ al día, o sea, $200 \mathrm{mg}$ cada 6 h vía oral. En pediatría, aún no hay consenso en la dosificación óptima, existiendo como referencia lo reportado por el Hospital de Niños St Jude (Memphis, E.U.A.), quienes recomiendan una dosis con un corte ajustado por peso: en niños con menos de 34 kilos sugieren una dosis de 18 a $24 \mathrm{mg} / \mathrm{kg} /$ día, oral dividida en 4 dosis y niños con más de 34 kilos, $800 \mathrm{mg}$ diarios divididos en 4 dosis $^{2}$. Ese centro utiliza habitualmente la medición de concentraciones plasmáticas (CPs) a los 4 o 7 días de inicio de PSC, determinando una CP igual o mayor a $0,7 \mu \mathrm{g} / \mathrm{mL}$ para profilaxis y CP igual o mayor a $1,25 \mu \mathrm{g} / \mathrm{mL}$ para tratamiento, criterio acorde a lo comunicado en otros estudios donde se encuentra una correcta correlación con la respuesta al antifúngico ${ }^{2-4}$. Se ha demostrado la seguridad y eficacia de PSC en la
Facultad de Medicina, Universidad de Chile, Santiago, Chile. Departamento de Pediatría y Cirugía Infantil Oriente, Hospital Dr. Luis Calvo Mackenna, Unidad de Investigación (RV, MES, JPT). Hospital Dr. Luis Calvo Mackenna, Santiago, Chile. Unidad de Trasplante de Médula Ósea (JP, PC).

Unidad de Farmacia (PG, MB, JM).

Los autores declaran no tener conflictos de interés.

No hubo financiamiento para la realización de este trabajo

Recibido: 21 de agosto de 2017 Aceptado: 7 de diciembre de 2017

Correspondencia a: Jorge Morales Vallespín jmorales@calvomackenna.cl 
administración en pacientes pediátricos; sin embargo, la farmacocinética en niños bajo 13 años de edad es aún poco conocida ${ }^{5}$.

Una de las dificultades que presenta PSC es que su farmacocinética se ve afectada por varios factores: la dieta, medicamentos, alteraciones gastrointestinales, mucositis, frecuencia de las dosis y estado prandial, lo que se refleja en su biodisponibilidad ${ }^{6}$. Esto determina un especial desafío en la profilaxis y/o tratamiento de la IFI en niños, siendo necesario aumentar el conocimiento relacionado al uso de este medicamento y las CPs alcanzadas en la población pediátrica, que tiene una farmacocinética más compleja asociada a la maduración de sus sistemas hepáticos y renales que alteran las CPs de los medicamentos ${ }^{2-6}$.

El objetivo de nuestro estudio fue describir la experiencia de monitoreo de las CPs de PSC en pacientes pediátricos inmunocomprometidos con IFI y determinar si las dosis recomendadas alcanzan CPs efectivas en profilaxis $(\geq 0,7 \mu \mathrm{g} / \mathrm{mL})$ y tratamiento $(\geq 1,25 \mu \mathrm{g} / \mathrm{mL})$.

\section{Material y Método}

Estudio descriptivo, retrospectivo, en niños internados por sospecha o confirmación de IFI entre enero de 2012 y octubre de 2016, en la Unidad de Trasplante de Médula Ósea y Oncología del Hospital Dr. Luis Calvo Mackenna (HLCM). Con la autorización del Director del hospital, los datos fueron obtenidos desde la ficha clínicas, fichas de seguimiento farmacoterapéutico (documentos de farmacia clínica) y el sistema de laboratorio clínico en línea del HLCM (SYSLAB®).

Estudio descriptivo retrospectivo, en pacientes pediátricos con indicación de posaconazol entre enero de 2012 y octubre de 2016

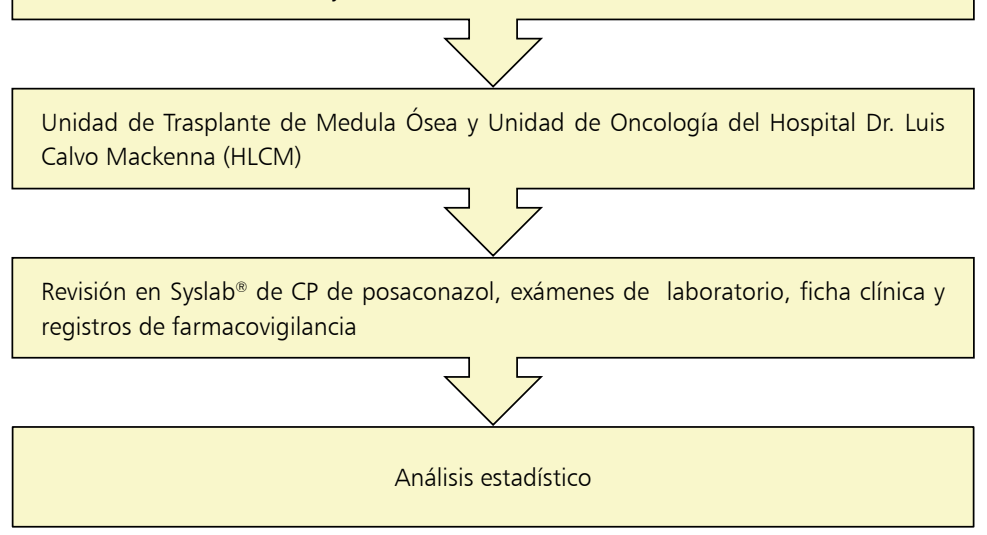

Figura 1. Metodología.
Se incluyeron aquellos casos que recibieron PSC en suspensión para profilaxis o tratamiento de IFI probada o probable, de acuerdo a los criterios del consenso EORTC/MSG (Tabla 1) y excluyéndose a los niños que cambiaron antifúngico durante el episodio de interés. El perfil de seguridad fue obtenido y analizado de acuerdo a farmacovigilancia del uso de PSC, que corresponde a la evaluación y seguimiento farmacoterapéutico del equipo de farmacia clínica en conjunto con el equipo clínico, a través del algoritmo de Naranjo cuando correspondía.

La medición de CPs basales de PSC se realizó con cromatografía liquida de alta eficacia, $30 \mathrm{~min}$ antes de la administración de la dosis a los 4 o 7 días de inicio de PSC y en pacientes en seguimiento por el riesgo de IFI, por el equipo de enfermería de cada unidad (Figura 1).

\section{Resultados}

Durante el período del estudio hubo seis pacientes (Tabla 2) (cuatro indicaciones de profilaxis y cuatro tratamientos) con leucemia linfoblástica aguda (LLA), que cumplieron los criterios de ingreso, con un total de 78 determinaciones de CPs basales para PSC. Se excluyeron cinco pacientes por cambio de agente triazólico a voriconazol por mayor especificidad de tratamiento para aspergilosis; esto ocurrió en la primera semana de tratamiento. La mediana de dosis de PSC administrada en suspensión fue de 12,5 y $18,8 \mathrm{mg} / \mathrm{kg}$ /día para profilaxis y tratamiento, resultando en CP mediana de 0,97 y $1,8 \mu \mathrm{g} /$ $\mathrm{mL}$, respectivamente (Tabla 3 ).

Para los episodios de profilaxis se registraron $40 / 67$

Tabla 1. Datos demográficos de los pacientes pediátricos hospitalizados entre enero de 2012 y octubre de 2016 con uso de posaconazol como profilaxis y tratamiento

\begin{tabular}{|ll}
\hline Pacientes (n) & 6 \\
\hline$N^{\circ}$ total de mediciones de CPs & 78 \\
\hline Diagnóstico de base ( $\mathrm{n}$ ) & LLA (6/6) \\
\hline Cultivos aislados ( $\mathrm{n})$ & $\begin{array}{l}\text { Mucor sp (4) } \\
\text { Aspergillus spp (1) } \\
\text { Malassezia furfur (1) } \\
\text { Rhizopus spp (1) }\end{array}$ \\
\hline Edad-mediana (años) [rango] & 12 [5-16] \\
\hline Sexo masculino (\%) & 3 (50) \\
\hline CP: concentración plasmática. & \\
\hline
\end{tabular}




\begin{tabular}{|c|c|c|c|c|}
\hline & \multicolumn{2}{|c|}{ Profilaxis } & \multicolumn{2}{|c|}{ Tratamiento } \\
\hline & $\mathrm{CP}<0,7 \mu \mathrm{g} / \mathrm{mL}$ & $\mathrm{CP} \geq 0,7 \mu \mathrm{g} / \mathrm{mL}$ & $\mathrm{CP}<1,25 \mu \mathrm{g} / \mathrm{mL}$ & $\mathrm{CP} \geq \mu \mathrm{g} / \mathrm{mL} 1,25$ \\
\hline Mediciones de CP (n:78) & 27 & 40 & 6 & 5 \\
\hline $\mathrm{CP}$ promedio $\mu \mathrm{g} / \mathrm{mL}$ & $0,47 \pm 0,15$ & $1,05 \pm 0,34$ & $0,93 \pm 0,18$ & $1,90 \pm 0,62$ \\
\hline $\mathrm{CP}$ mediana $\mu \mathrm{g} / \mathrm{mL}$ & $0,54(0,13-0,66)$ & $0,97(0,34-2,31)$ & $0,93(0,18-1,22)$ & $1,8(0,62-2,94)$ \\
\hline Dosis promedio mg/kg/día & $19,2 \pm 16,8$ & $16,0 \pm 14,7$ & $23,3 \pm 15,8$ & $18,0 \pm 18,8$ \\
\hline Dosis mediana mg/kg/día & $9,4(4,7-55,2)$ & $12,5(4,7-55,2)$ & $18,8(12,5-55,2)$ & $18,8(5,29-25)$ \\
\hline
\end{tabular}

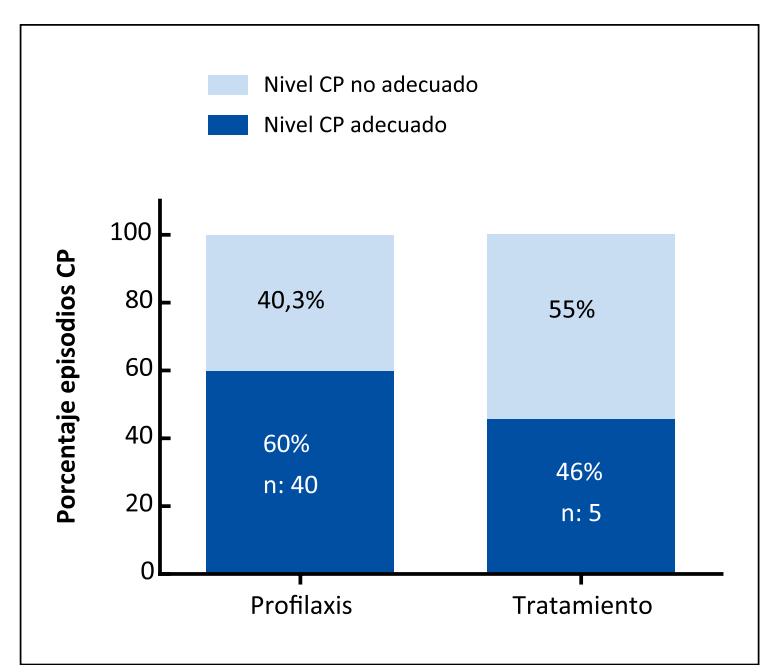

Figura 2. Total de concentraciones plasmáticas de posaconazol $(\mathrm{n}=78)$. CP: Concentraciones plasmáticas.

$(60 \%)$ determinaciones en CP promedio $\geq 0,70 \mu \mathrm{g} / \mathrm{mL}$ recibiendo una mediana de dosis de $12,5 \mathrm{mg} / \mathrm{kg} /$ día. Mientras que, para el tratamiento, 5/11 (46\%) determinaciones presentaron $\mathrm{CP} \geq 1,25 \mu \mathrm{g} / \mathrm{mL}$, recibiendo una mediana de dosis de $18 \mathrm{mg} / \mathrm{kg} /$ día (Tabla 2, Figura 2). La dosis máxima de PSC utilizada fue de $800 \mathrm{mg} /$ día (en pacientes mayores a 13 años). Los resultados para profilaxis y tratamiento mostraron una marcada variabilidad interpaciente e intrapaciente (Figuras 3 y 4 ).

Dos niños de los seis presentaron neuropatía grave debido a la interacción entre vincristina y PSC (Figura 5), los que requirieron tratamiento con piridoxina $\mathrm{y}$ mejoraron su condición. No se observó reacción adversa alguna en esta cohorte, ni nefrotoxicidad o hepatotoxicidad, sólo un incremento reversible de las transaminasas hepáticas, sin repercusión clínica, y las alteraciones electrolíticas observadas no fueron atribuidas al uso de PSC.

\begin{tabular}{|c|c|}
\hline Mediana dosis profilaxis (mg/kg/día) [rango] & $12,5(4,7-55,2) n=67$ \\
\hline Mediana dosis tratamiento (mg/kg/día) [rango] & $18,8(12,1-55,2) n=11$ \\
\hline Mediana CP en profilaxis $(\mu \mathrm{g} / \mathrm{mL})$ [rango] & $0,97(0,34-2,31)$ \\
\hline Mediana CP en tratamiento $(\mu \mathrm{g} / \mathrm{mL})$ [rango] & $1,8(0,62-2,94)$ \\
\hline Adecuada CP en profilaxis (CP > 0,7) (\%) & $40(60)$ \\
\hline Adecuada CP en tratamiento (CP > 1,25) (\%) & $5(46)$ \\
\hline
\end{tabular}

Tabla 3. Dosis de posaconazol empleadas en profilaxis y tratamiento de infecciones fúngicas invasoras y concentraciones plasmáticas alcanzadas en 6 pacientes pediá-

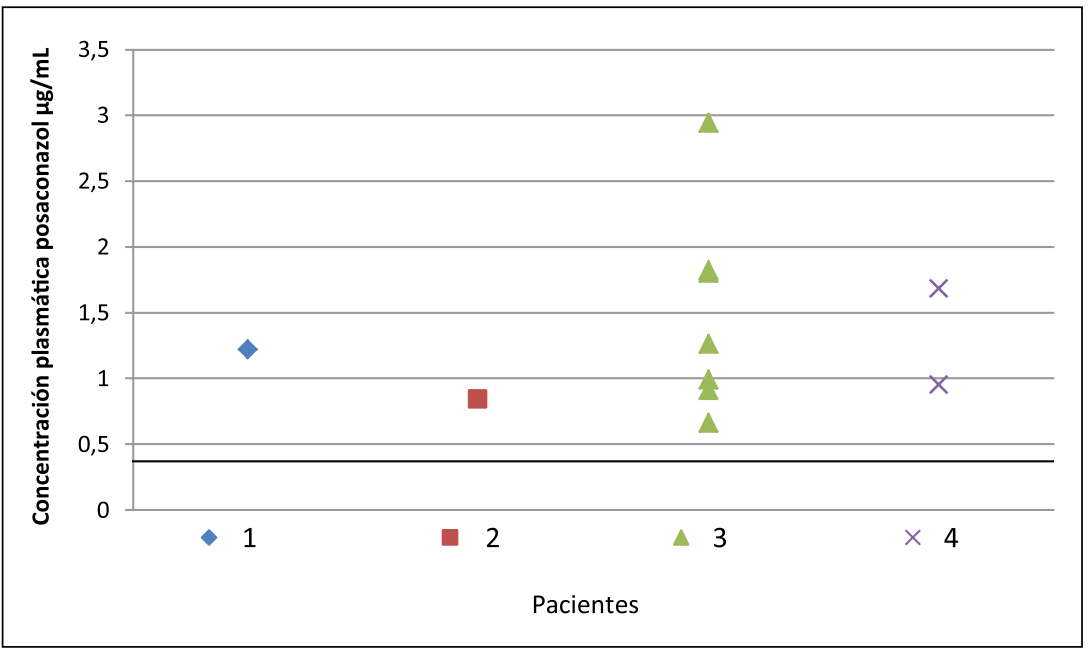

Figura 3. Concentraciones plasmáticas de posaconazol por pacientes en tratamiento $(n=4)$. Nota: Se solapa una CP del paciente 3, por eso se cuentan 10 en vez de 11 niveles. 


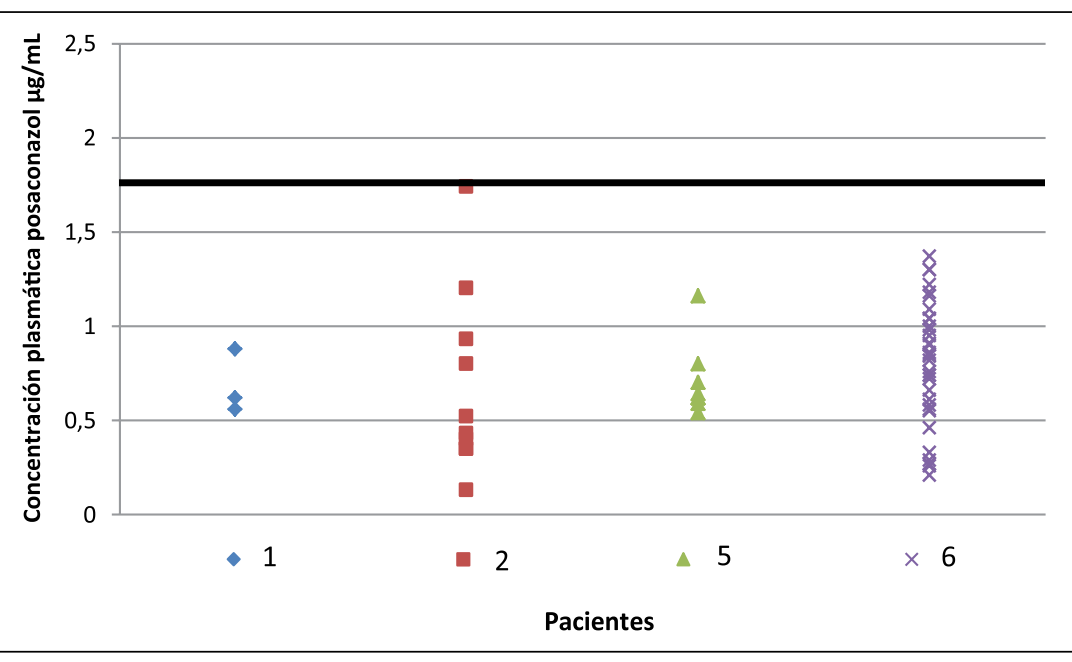

Figura 4. Concentraciones plasmáticas de posaconazol por pacientes en profilaxis $(n=4)$.

Figura 5. Reacciones adversas asociadas a la interacción de vincristina-posaconazol $(n=6)$. en los pacientes bajo 13 años de edad, incrementando el riesgo de morbilidad de IFI.

Diversos estudios han descrito la monitorización de PSC y las relaciones de dosis en niños. Krishna y cols ${ }^{7}$., realizaron un análisis secundario comparando los datos de CPs de PSC en 12 niños (edad mediana: 14 años, rango: 8-17). Como conclusión, las CPs de PSC fueron similares en pacientes jóvenes y adultos. Los objetivos secundarios fueron analizar la eficacia en la población juvenil y comparar los perfiles de seguridad de PSC para pacientes jóvenes y adultos, el que fue seguro y bien tolerado; además, tuvo perfiles de eventos adversos similares tanto para pacientes jóvenes como adultos.

En nuestro estudio, la mediana de edad (12 años) fue similar a la mediana de edad observada por Lehrnbecher y cols. ${ }^{8}$, y menor que en el estudio de Krishna y cols. La mediana de dosis en nuestro estudio $(12,5 \mathrm{mg} / \mathrm{kg} /$ día) fue similar a la mediana de la dosis en el estudio realizado por Krishna y cols., pero menor que la reportada por Lehrnbecher y cols. Otros estudios retrospectivos (Doring, McMahon, Mathew) ${ }^{9-11}$ de análisis de CP de PSC en pacientes inmunocomprometidos pediátricos mostraron que con medianas de dosis de $12-12,5 \mathrm{mg} / \mathrm{kg} /$ día se lograban CPs menores a las recomendadas para profilaxis. McMahom y cols., por ejemplo encontraron en su estudio que en $46 \%$ de los pacientes tratados con una dosis mediana de $12,5 \mathrm{mg} / \mathrm{kg} /$ día se lograron CPs $>$ a 0,7 y que en $69,2 \%$ de los paciente en tratamiento con PSC no se lograron CPs $>1,25$ con una mediana de dosis de 20,5. Por otra parte, Mathew y cols., en un análisis de 32 pacientes observaron que sólo en $50 \%$ de los pacientes tratados con una mediana de dosis de $17,5 \mathrm{mg} / \mathrm{kg} /$ día se lograron $\mathrm{CP}>0,7$ y que la dosis mediana para lograr CPs adecuadas para profilaxis fue de $22,8 \mathrm{mg} / \mathrm{kg} / \mathrm{día}$. En otro estudio multicéntrico realizado por De Pauw y cols. ${ }^{12}$, fase III, abierto, de PSC en 194 pacientes adultos, con la excepción de un paciente pediátrico, todos los pacientes recibieron una dosis de mantención de $800 \mathrm{mg}$ diarios de PSC en suspensión en dosis dividida. La dosis media para los pacientes pediátricos fue de $16,5 \mathrm{mg} / \mathrm{kg} /$ día, con CPs de PSC similares en pacientes pediátricos y adultos, con valores medios de $0,78 \mu \mathrm{g} / \mathrm{mL}$ y $0,82 \mu \mathrm{g} / \mathrm{mL}$, respectivamente. Además, Lehrnbecher y cols., informaron una mediana de dosis de $21 \mathrm{mg} / \mathrm{kg} /$ día (rango: 4,8-33,3 mg/kg/ día) en 15 niños (edad mediana: 10 años) que recibieron PSC para tratar infecciones fúngicas. Las CPs de PSC no se informaron en este estudio ${ }^{8}$. Esta información se correlaciona con los datos obtenidos en el presente estudio, con una mediana de dosis para nuestros pacientes pediátricos de $12,5(4,7-55,2)$ y $18,8(5,29-25) \mathrm{mg} / \mathrm{kg} /$ día, alcanzando CPs promedio de $0,97(0,34-2,31) \mu \mathrm{g} / \mathrm{mL}$ y $1,8(0,62-2,94)$ $\mu \mathrm{g} / \mathrm{mL}$, para profilaxis y tratamiento, respectivamente.

Sobre la base de estas CPs, los pacientes bajo 13 años de edad parecen requerir una dosis más alta de la que es 
utilizada habitualmente para alcanzar niveles que pueden ser efectivos para la IFI. Aunque las concentraciones objetivo de PSC no se han establecido definitivamente, varios estudios han demostrado la relación entre la respuesta clínica y la $\mathrm{CP}$ de este antifúngico ${ }^{12-14}$. En un estudio de investigación abierto, Walsh y cols. ${ }^{15}$, informaron que los pacientes con aspergilosis invasora refractaria que tenían una $\mathrm{CP}$ promedio de PSC de $1,25 \mu \mathrm{g} / \mathrm{mL}$ tuvieron una mejor respuesta clínica (75\%) que aquellos con una $\mathrm{CP}$ promedio de $0,13 \mu \mathrm{g} / \mathrm{mL}$ (24\%). En el trabajo de Dolton y cols. ${ }^{16}$, se revisó la efectividad clínica de PSC en 86 pacientes donde (72/86) pacientes recibieron PSC como profilaxis y pudieron determinar que $12 / 72$ (17\%) tuvieron infección por hongos y tenían CP promedio de $0,289 \mu \mathrm{g} /$ $\mathrm{mL}$, que fue significativamente menor que aquellos que no desarrollaron infecciones invasoras (CP promedio: $0,485 \mu \mathrm{g} / \mathrm{mL})(\mathrm{p}<0,05)$. En cambio, 14/86, pacientes recibieron PSC como tratamiento, de quienes 4/14 (29\%) fracasaron en la terapia y tuvieron $\mathrm{CP}$ promedio de 0,436 $\mu \mathrm{g} / \mathrm{mL}$, lo que fue significativamente menor que la $\mathrm{CP}$ de los que respondieron al tratamiento (CP promedio: 0,955 $\mu \mathrm{g} / \mathrm{mL})(\mathrm{p}=0,19)$. En relación a nuestros resultados, si bien 60 y $46 \%$ están sobre el corte óptimo para profilaxis y tratamiento, aún así no se reportó recaída o empeoramiento del cuadro clínico para los pacientes observados. Es importante seguir monitorizando para optimizar el tratamiento y tener CP para una dosificación segura y eficaz en cada paciente.

En niños, Kim Vanstraelen y cols. ${ }^{17}$, revisaron la dosificación de PSC profiláctica en 14 pacientes (edad promedio: 6,7 años) a dosis de $120 \mathrm{mg} / \mathrm{mt}^{2}$ cada $8 \mathrm{~h}$ (equivalente a $12 \mathrm{mg} / \mathrm{kg} /$ día), los que alcanzaron un promedio de CP de $0,85 \mu \mathrm{g} / \mathrm{mL}$, considerado óptimo para la profilaxis, donde la administración de PSC fue con un protocolo para evitar interferencias con otros medicamentos y/o comidas ${ }^{6,11,19}$. Sin embargo, en una revisión de casos pediátricos de $\mathrm{V}$. Bernardo y cols. ${ }^{2}$, se revisaron 33 pacientes (mediana de edad: 11,5 años) y encontraron que con una media de dosis de $20 \mathrm{mg} / \mathrm{kg} /$ día cada $6 \mathrm{~h}$, la CP fue mayor a $0,7 \mu \mathrm{g} / \mathrm{mL}$ (como tratamiento o profilaxis) en 21 pacientes. Aquellos que tuvieron una $\mathrm{CP}$ menor a $0,7 \mu \mathrm{g} / \mathrm{mL}$, habían recibido una dosificación de $12 \mathrm{mg} / \mathrm{kg} /$ día en 12 pacientes. Este trabajo mostró resultados muy similares a los obtenidos en nuestra experiencia de utilizar dosis mayores a $12 \mathrm{mg} / \mathrm{kg} /$ día, encontrándose dosis similares de $20 \mathrm{mg} / \mathrm{kg} /$ día para obtener CPs mayores de $0,7 \mu \mathrm{g} / \mathrm{mL}$. Además, los estudios clínicos controlados revelaron que una CP de PSC de 0,7 $\mu \mathrm{g} / \mathrm{mL}$ o mayor es necesaria para minimizar la incidencia de infecciones fúngicas, lo que es considerado como la CP mínima necesario para profilaxis. Usando estos datos, seleccionamos $0,7 \mu \mathrm{g} / \mathrm{mL}$ como nuestra concentración objetivo para profilaxis y al mismo tiempo $\mathrm{CP}>1,25$ $\mu \mathrm{g} / \mathrm{mL}$ para tratamiento, que ha recomendado el grupo pediátrico de Bernardo y cols.
Se ha demostrado que varios factores afectan a la farmacocinética de PSC, incluyendo el $\mathrm{pH}$ gástrico, la frecuencia de dosificación, el estado prandial, el momento del consumo de alimentos, la motilidad gástrica, la enfermedad subyacente y la mucositis ${ }^{13,18-19}$. La diarrea ha sido identificada como un factor correlacionado con la reducción de la absorción de PSC, existiendo una variabilidad interpaciente significativa en los parámetros farma$\operatorname{cocinéticos}^{18-19}$. El monitoreo farmacológico terapéutico puede tener un papel crucial en el cuidado de pacientes que reciben PSC, especialmente en aquellos con sospecha de fracaso terapéutico, infecciones debidas a microorganismos resistentes a fármacos, mucositis, malabsorción y en aquellos que no pueden tomar PSC con alimentos ricos en grasas ${ }^{18-20}$. Otros estudios han demostrado que muchos adultos, incluidos los pacientes neutropénicos, tratados con PSC no alcanzan las concentraciones plasmáticas deseadas Bryant y cols ${ }^{19}$.

En nuestra experiencia, PSC fue bien tolerado; sin embargo, dos niños de los seis presentaron neuropatía grave debido a la interacción medicamentosa con vincristina, requiriendo tratamiento con piridoxina, con respuesta clínica favorable. Esta interacción se encuentra descrita y es reversible, pero se debe tomar en consideración para la administración de ambos fármacos. No existe una relación definida entre las CPs de PSC y el efecto adverso de la neuropatía asociada al alcaloide de la vinca ${ }^{21,22}$. Con la anterior consideración, elaboramos un protocolo de administración en el hospital, en el que el antifúngico se suspende $24 \mathrm{~h}$ antes de administrar la vincristina y se reanuda sólo 48 horas después de aplicada la quimioterapia, en aquellas circunstancias que han de usarse ambos fármacos (Nota del Editor: Documento disponible mediante solicitud a los autores).

Otro efecto adverso común fue un aumento transitorio en los valores de función hepática que se resolvieron mientras el tratamiento continuaba y no tuvieron relevancia clínica. Este resultado es concordante con los de otros estudios que evalúan la seguridad de PSC en pacientes pediátricos ${ }^{2,12,21-22}$. Lehrnbecher y cols., comunicaron que $73 \%$ de sus pacientes experimentaron algún evento adverso, como fiebre, náuseas y/o vómitos, dolor abdominal, diarrea, cefalea, erupciones cutáneas y elevación de las pruebas de función hepática. Los aumentos frecuentes en los parámetros hepáticos de laboratorio se atribuyeron a los múltiples medicamentos concomitantes que recibieron estos pacientes. Debido a que la monitorización terapéutica del fármaco no se realizó en este estudio, no se pueden hacer asociaciones con respecto a la incidencia de toxicidad hepática y CP de PSC. De manera similar, en el estudio realizado por Krishna y cols., los eventos adversos más comunes fueron náuseas, vómitos y cefalea. Además, dos pacientes (16\%) presentaron resultados elevados en las pruebas de función hepática. Sin embargo, se consideró 
que ambas elevaciones no estaban relacionadas con el tratamiento con $\mathrm{PSC}^{8}$.

\section{Limitaciones del trabajo}

Por su carácter retrospectivo presenta una desventaja intrínseca; el bajo número de pacientes no representa una buena muestra para una determinación más claras de reacciones adversas y evaluaciones clínicas relevantes con respecto a las interacciones. No obstante, es de gran interés científico esta revisión de antifúngicos y $\mathrm{CP}$ de una cohorte de pacientes locales, considerando la relevancia del tema y lo poco investigado en nuestro medio, más aún, en el ámbito pediátrico.

En conclusión, nuestros resultados se ajustan a lo recomendado internacionalmente para la dosificación de PSC, pero se requiere una monitorización individualizada para mantener adecuadas CPs, ya que nuestra presentación de formulación tiene ciertas limitantes en cuanto a absorción e interacción con alimentos y es necesario reforzar la correcta administración del medicamento.

Otro aspecto a considerar es evaluar los riesgos de interacciones medicamentosas para cada paciente y considerar la suspensión o ajuste de dosis en la administración conjunta para alcaloides de la vinca e inmunosupresores. A pesar de no encontrar ninguna reacción adversa, creemos que es relevante recomendar la monitorización periódica de niños en tratamiento con PSC.

Agradecimientos. A Sofía Canals y Manuel Azocar por la colaboración en la etapa inicial del estudio y a Carolina Salas por su ayuda con la determinación de concentraciones plasmáticos, Iván Gajardo por su colaboración en las figuras del trabajo y al Equipo de Farmacia del HLCM por su apoyo incondicional.

\section{Resumen}

Introducción: En pediatría no existe consenso en la dosificación de posaconazol (PSC) para profilaxis y tratamiento de la infección fúngica invasora (IFI), usándose la medición de concentraciones plasmáticas (CPs) del fármaco. Objetivo: Describir la experiencia de monitoreo de las CPs de PSC en niños inmunocomprometidos con IFI y determinar si las dosis recomendadas alcanzan CPs efectivas en profilaxis $(\geq 0,7 \mu \mathrm{g} / \mathrm{mL})$ y tratamiento $(\geq 1,25$ $\mu \mathrm{g} / \mathrm{mL})$. Método: Análisis retrospectivo en niños que recibieron PSC suspensión como profilaxis o tratamiento entre enero de 2012 y octubre de 2016, en las unidades de Oncología y Trasplante de Médula Ósea del Hospital Calvo Mackenna. Resultados: 78 CPs en seis pacientes (4 indicaciones de profilaxis y 4 tratamientos) fueron revisados. La mediana de dosis de PSC fue de 12,5 y 18,8 $\mathrm{mg} / \mathrm{kg} / \mathrm{d}$ para profilaxis y tratamiento, respectivamente, resultando CP mediana de 0,97 y $1,8 \mu \mathrm{g} / \mathrm{mL}$, respectivamente. En profilaxis, se registraron 40/67 (60\%) con CP $\geq 0,70 \mu \mathrm{g} / \mathrm{mL}$ recibiendo una mediana de dosis de 12,5 $\mathrm{mg} / \mathrm{kg} / \mathrm{d}$. Mientras que para el tratamiento: 5/11 (46\%), presentaron $\mathrm{CP} \geq 1,25 \mu \mathrm{g} / \mathrm{mL}$, recibiendo una mediana de dosis de $18 \mathrm{mg} / \mathrm{kg} / \mathrm{d}$. Conclusión: Nuestros resultados se ajustan a lo recomendado para la dosificación de PSC, pero evidencian una necesidad de realizar una monitorización individualizada para mantener adecuadas CPs.

\section{Referencias bibliográficas}

1.- Vallejo J C, Ruiz-Camps I. Infección fúngica invasora en los pacientes hematológicos. Enferm Infecc Microbiol Clin 2012; 30 (9): 572-9.

2.- Bernardo V, Cross S J, Crews K R, Flynn P M, Hoffman J M, Knapp K M, et al. Posaconazole therapeutic drug monitoring in pediatric patients and young adults with cancer. Ann Pharmacother 2013; 47 (7-8): 976-83. doi: 10.1345/aph.1R775.

3.- Döring M, Blume O, Haufe S, Hartmann U, Kimmig A, Schwarze C P, et al. Comparison of itraconazole, voriconazole, and posaconazole as oral antifungal prophylaxis in pediatric patients following allogeneic hematopoietic stem cell transplantation. Eur J Clin Microbiol Infect Dis 2014; 33: 629-8.

4.- Mattiuzzi G, Yilmaz M, Kantarjian H, Borthakur G, Konopleva M, Jabbour E, et al. Pharmacokinetics of posaconazole prophylaxis of patients with acute 0myeloid leukemia. J Infect Chemother 2015; 21: 663-7.

5.- BN Gross, Ihorst G, Jung M, Wäsch R, Engelhardt M. Posaconazole therapeutic drug monitoring in the real-life setting: a single-center experience and review of the literature. Pharmacotherapy 2013; 33 (10): 1117-25.

6.- Gwee A, Cranswick N, Curtis N. Posaconazole: promising but problematic in practice in pediatric patients. Pediatr Infect Dis J 2015; 34 (6): 604-6.

7.- Krishna G Sansone-Parsons A, Martinho M, Kantesaria B, Pedicone L. Posaconazole plasma concentrations in juvenile patients with invasive fungal infection. Antimicrob Agents Chemother 2007; 51: 812-8.

8.- Lehrnbecher T, Attarbaschi A, Duerken M, Garbino J, Gruhn B, Kontny U, et al. Posaconazole salvage treatment in paediatric patients: a multicentre survey. Eur J Clin Microbiol Infect Dis 2010; 29: 1043-5.
9.- Döring M, Cabanillas Stanchi K M, Klinker H, Eikemeier M, Feucht J, Blaeschke F, et al. Posaconazole plasma concentrations in pediatric patients receiving antifungal prophylaxis during neutropenia. Med Mycol 2017; 55: 375-84.

10.- MacMahon J, Théoret $Y$, Autmizguine J, Bittencourt H, Tapiéro B, Ovetchkine P. Posaconazole plasma monitoring in immunocompromised children. J Pediatric Infect Dis Soc 2017; 6 (4):389-92. doi: 10.1093/jpids/piw087.

11.- Mathew S, Kussin M, Liu D, Pozotrigo M, Seyboth B, Thackray J, et al. Retrospective analysis of posaconazole suspension dosing strategies in a pediatric oncology population: single-center experience. J Pediatric Infect Dis Soc 2017; 6 (3): e149-51.

12.- De Pauw B, Walsh T J, Donnelly P, Stevens D A, Edwards J E, Calandra T, et al. Revised definitions of invasive fungal disease from the European Organization for Research and Treatment of Cancer/Invasive Fungal Infections 
Cooperative Group and the National Institute of Allergy and Infectious Diseases Mycoses Study Group (EORTC/MSG) Consensus Group. Clin Infect Dis 2008; 46: 1813-21.

13.- Hussaini T Rüping M J, Farowski F, Vehreschild J J, Cornely O A. Therapeutic drug monitoring of voriconazole and posaconazole. Pharmacotherapy 2011; 31 : 214-25.

14.- Andes D, Pascual A, Marchetti O. Antifungal therapeutic drug monitoring: established and emerging indications. Antimicrob Agents Chemother 2009; 53: 24-34.

15.- Walsh T J, Raad I, Patterson T F, Chandrasekar P, Donowitz G R, Graybill R, et al. Treatment of invasive aspergillosis with posaconazole in patients who are refractory to or intolerant of conventional therapy: An externally controlled trial. Clin Infect Dis 2007; 44: 2-12.
16.- Dolton M J, Ray J E, Chen S C, Ng K, Pont L, McLachlan A J. Multicenter study of posaconazole therapeutic drug monitoring: exposure-response relationship and factors affecting concentration. Antimicrob Agents Chemother 2012; 56: 5503-10.

17.- Vanstraelen K, Colita A, Bica A M, Mols R, Augustijns P, Peersman N, et al. Pharmacokinetics of posaconazole oral suspension in children dosed according to body surface area. Pediatr Infect Dis J 2016; 35 (2): 183-8.

18.- Jang S. Colangelo P M, Gobburu J V. Exposure-response of posaconazole for prophylaxis of invasive fungal infections: evaluation of the need for adjusting doses based on plasma drug concentrations. Clin Pharmacol Ther 2010; 88: 115-9.

19.- Bryant A M, Slain D, Cumpston A, Craig M. A post-marketing evaluation of posaconazole plasma concentrations in neutropenic patients with haematological malignancy receiving posaconazole prophylaxis. Int J Antimicrob Agents 2011; 37: 266-9.

20.- Kohl V, Müller C, Cornely O A, Abduljalil K, Fuhr U, Vehreschild J J, et al. Factors influencing pharmacokinetics of prophylactic posaconazole in patients undergoing allogeneic stem cell transplantation. Antimicrob Agents Chemother 2010; 54: 207-12.

21.- Common Terminology Criteria for Adverse Events v3.0 (CTCAE). Available at: http://ctep. cancer.gov/protocolDevelopment/electronic applications/docs/ctcaev3.pdf

22.- Eiden C, Palenzuela G, Hillaire-Buys D, Margueritte G, Cociglio M, Hansel-Esteller $\mathrm{S}$, et al. Posaconazole-increased vincristine neurotoxicity in a child: a case report. J Pediatr Hematol Oncol 2009; 31 (4): 292-5. 